\title{
Recurrence Factors and Characteristic Trends of Papillary Thyroid Cancer over Three Decades
}

\author{
Waralee Chatchomchuan (D), Yotsapon Thewjitcharoen (D), Krittadhee Karndumri, \\ Sriurai Porramatikul, Sirinate Krittiyawong, Ekgaluck Wanothayaroj, \\ Somboon Vongterapak, Siriwan Butadej, Veekij Veerasomboonsin, \\ Auchai Kanchanapitak, Rajata Rajatanavin, and Thep Himathongkam
}

Diabetes and Thyroid Center, Theptarin Hospital, Bangkok, Thailand

Correspondence should be addressed to Waralee Chatchomchuan; waralee.md@gmail.com

Received 3 April 2021; Accepted 29 April 2021; Published 11 May 2021

Academic Editor: Andrea Palermo

Copyright (c) 2021 Waralee Chatchomchuan et al. This is an open access article distributed under the Creative Commons Attribution License, which permits unrestricted use, distribution, and reproduction in any medium, provided the original work is properly cited.

\begin{abstract}
Background. The prevalence of thyroid cancer is rising worldwide. Although thyroid cancer has a favorable prognosis, up to $20 \%$ of patients experienced recurrent disease during the follow-up period. The present study aimed to examine the trend of incidence and factors associated with recurrence and outcomes of papillary thyroid cancer (PTC) in Thai patients over the last 30 years. Methods. We reviewed the clinical data of all patients with PTC who were treated between 1987 and 2019 at Theptarin Hospital. Clinical characteristics, epidemic trend, factors associated with the persistence/recurrence of the disease, overall disease-specific survival rate, and overall disease-free survival rate were analysed. Results. A total of 235 patients with PTC who were registered between 1987 and 2019 were reviewed. The mean age was $42.5 \pm 14.3$ years, with a mean follow-up of 9.5 years. Papillary thyroid microcarcinoma (PTMC) was consistently increased and accounted for $21.4 \%$ (50/235) of total cases. The American Thyroid Association (ATA) risk stratification was high in $24 \%$ of all PTMCs in the last decade, and $16.0 \%$ of these patients experienced local recurrence during the follow-up period. Coexistence with Hashimoto's thyroiditis (HT) was found in one-fifth of the patients with PTC and was correlated with a low recurrence rate (HR: $0.16, P=0.013$ ). Only age $\geq 55$ years associated with the persistence/ recurrence of the disease. The overall disease-free survival and disease-specific survival rates were $77.4 \%$ and $98.3 \%$, respectively. Conclusions. The prognosis of PTC is generally considered favorable. However, approximately one-fourth of patients with PTMC demonstrated more aggressive clinical behavior, particularly in the last decade of the study. Coexistence of HT contributed to a better prognosis.
\end{abstract}

\section{Introduction}

Thyroid cancer is the most common form of endocrine cancer, accounting for $3 \%$ of all new cancer cases in the United States $[1,2]$. Recently, an incidental finding of a small thyroid cancer, known as microcarcinoma, has gained considerable attention [3]. Papillary thyroid cancer (PTC) has generally been documented as an indolent, nonaggressive cancer with a low mortality rate [4]. However, several studies have reported a recurrence in approximately $20 \%$ of patients with this disease, in which nearly half of them were identified more than five years after the initial operation $[1,4]$. Although recurrence of the disease is not necessarily fatal, it inflicts lifelong and economic burdens to the patients.

Recently, the changing characteristics of papillary thyroid microcarcinoma (PTMC) were observed. PTMC was previously considered as having a good prognostic factor; however, additional studies have revealed otherwise on disease recurrence and metastasis [5]. It is unclear whether this occurrence is because of an increase in thyroid cancer incidence or changes in the disease itself. Recent guidelines have recommended PTMC treatment by lobectomy, unless it has aggressive characteristics [6]. However, a recent meta- 
analysis showed that less aggressive treatment in patients with PTMC increased the risk of recurrence compared with total thyroidectomy [7]. Identifying clinical characteristics for high-risk patients is essential for effective treatment of PTMC.

Racial disparity also affects cancer incidence and outcomes. Several studies revealed that thyroid cancer incidence was relatively high among Chinese, but lower in South Asian and non-Hispanic White [8-10]. A small cohort showed thyroid cancer tended to be more aggressive, with a higher rate of recurrence and death, in Filipinos compared to other races [11].

The present study aimed to explore the evolution of PTC in Thai patients regarding clinical characteristics, unfavorable risk factors, aggressiveness, and survival over a 30-year period.

\section{Materials and Methods}

2.1. Subjects and Data Collection. We reviewed the clinical data of all patients diagnosed with PTC who were registered between 1987 and 2019 at Theptarin Hospital, an endocrine center in Thailand. Exclusion criteria included age $<15$ years, non-PTC types (poorly differentiated thyroid cancer, medullary thyroid cancer, anaplastic thyroid cancer, and other differentiated thyroid cancers), incomplete data, and follow-up time less than six months (Figure 1).

All patients were categorized according to the American Thyroid Association (ATA) risk of recurrence stratification system and tumor-node-metastasis (TNM) staging criteria proposed by the American Joint Committee on Cancer (AJCC), $8^{\text {th }}$ edition [12]. Data on preoperative thyroid ultrasonography, type of operation, and the association of Hashimoto's thyroiditis (HT) were included for analysis. HT was confirmed by pathology. PTC with $\leq 1 \mathrm{~cm}$ diameter was defined as PTMC [6]. Serum thyroid-stimulating hormone (TSH) level, basal and stimulated thyroglobulin levels, neck ultrasonography, dosage and date of radioactive iodine ablation, and whole-body scan were monitored postoperatively. This study was approved by the Ethical Committee of Theptarin Hospital (EC number: 2/2019).

2.2. Treatment and Follow-Up. All patients were treated by surgeons performing more than 30 thyroid surgeries per year [13]. Disease monitoring and treatment were determined by the attending endocrinologists. Response to treatment was classified into four categories: excellent, indeterminate, biochemical incomplete, and structural incomplete response, according to the recent guidelines [6]. Time to recurrence was calculated from the day of initial surgery to the day of recurrence confirmed by cytological and/or pathological data. Persistence was defined as incomplete remission after the first surgery within one year.

2.3. Statistical Analysis. Statistical Package for the Social Sciences (version 21.0; IBM, New York, USA) was used for statistical analysis. Data were presented as means \pm standard deviations or medians \pm interquartile ranges (IQRs). Chi- square test was used to compare different categories. Differences in the mean between groups were analysed using a $t$-test or ANOVA test. Cox proportional hazards model was used to perform univariate and multivariate analyses to analyze the factors associated with recurrence. Potential risk predictors were age, sex, body mass index (BMI), ATA risk, coexistence of HT, tumor size, extrathyroidal extension, and multifocality. The disease-free survival rate was estimated using the Kaplan-Meier method and compared by using the log-rank test. All $P$ values were two-sided. $P<0.05$ was considered statistically significant.

\section{Results}

3.1. Clinical Characteristics and Trends of Thyroid Cancer. Table 1 summarizes the 235 cases that were included in the study. Approximately one-fifth of all cases of PTC were PTMC $(n=50,21.4 \%)$. Coexisting thyroid disease, including Graves' disease and HT, was found in 23 (9.8\%) and 46 $(19.6 \%)$ of the total patients, respectively. Patients with persistent/recurrent disease were older than those with remission, with the mean age of $44.7 \pm 16.3$ years vs. $41.8 \pm 13.6$ years, respectively. The median follow-up was 9.5 years (range: $0.5-31.3$ years).

In total, 201 (85.5\%) patients presented with a neck mass. Physical examination revealed thyroid tumors in 30 asymptomatic patients $(12.8 \%)$. Two patients $(0.9 \%)$ had abnormal neck ultrasonography. The remaining patients $(n=2,0.9 \%)$ had other initial presentations, including axillary lymph node enlargement and an incidental finding from a thyroidectomy specimen.

Of 235 patients, $81.7 \%$ underwent surgery at our hospital, while the rest were referred from other hospitals for further management after the initial operation. Total or near-total thyroidectomy was the most performed procedure $(n=226$, 96.2\%). Most patients $(n=231,98.3 \%)$ received at least one dose of postoperative radioactive iodine. Transient and permanent hypoparathyroidism were found in 35.3\% $(n=83)$ and $4.7 \%(n=11)$ of patients, respectively. Only $2.1 \%$ of patients experienced permanent recurrent laryngeal nerve injury. Most patients $(n=205,87.2 \%)$ received thyroid hormone suppression therapy, with serum TSH levels $<0.01 \mathrm{mU} / \mathrm{L}$.

Table 2 shows the prevalence rate of thyroid cancer divided by decade. The incidence of PTMC increased over the study time, from $15.2 \%$ in $1987-1996$ to $24.8 \%$ in 2007-2019, but without statistical significance, while the age, sex, BMI, and TNM stage revealed no differences. A significant increase of the high-risk ATA group among overall PTC patients was observed (13.0\% in $1987-1996$ vs. $34.4 \%$ in 2007-2019).

Additionally, a subgroup analysis of PTMC showed that $24 \%$ of all PTMCs belonged to the high-risk ATA group. This trend continued to rise over the study period. In the first decade of the study, all patients with PTMC were classified as low-risk ATA group, whereas in the second and third decades of the study, $4.7 \%$ and $7.2 \%$ of patients with PTMC were classified as high-risk ATA group, respectively (Figure 2). Furthermore, $16.0 \%$ of all PTMC patients developed a local recurrence. 


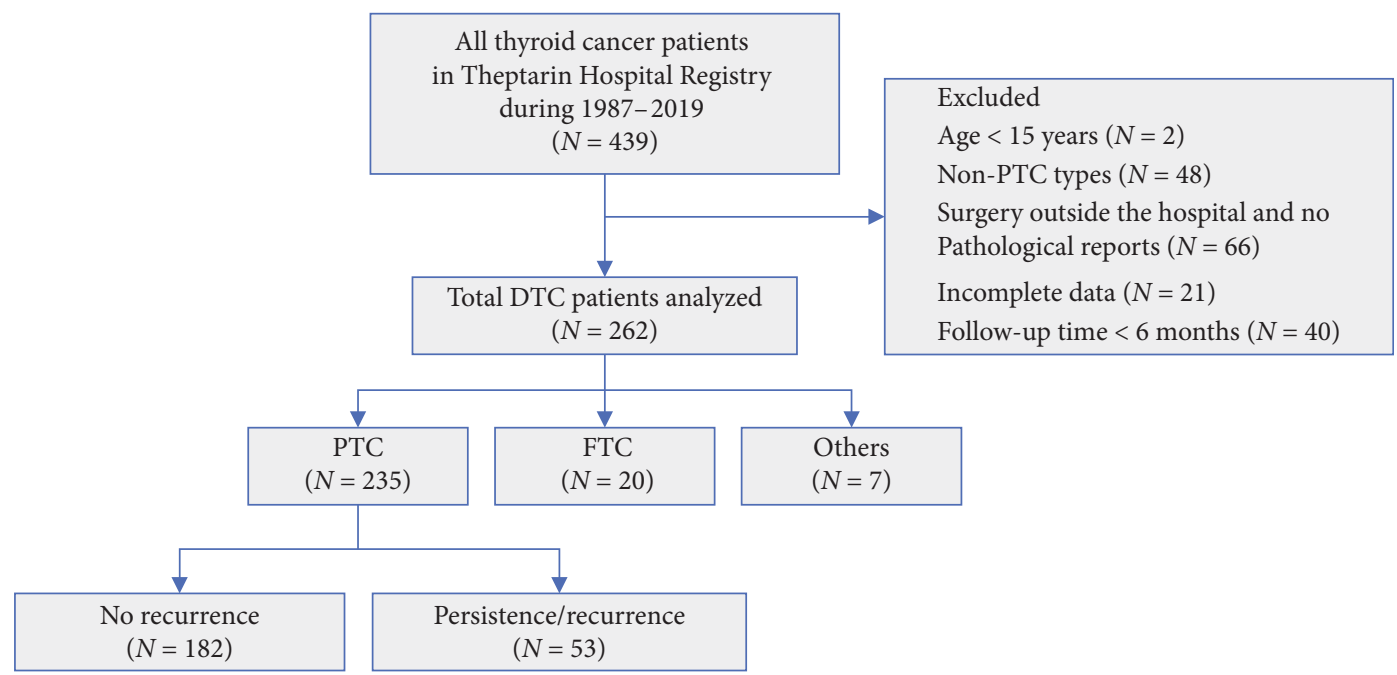

FIgURE 1: Flowchart depicting the protocol used in this study.

TABle 1: Demographic data of 235 papillary thyroid cancer patients.

\begin{tabular}{|c|c|c|c|c|}
\hline & Total $(n=235)$ & No recurrence $(n=182)$ & Persistence/recurrence $(n=53)$ & $P$ value \\
\hline Age at initial diagnosis (years) & $42.5 \pm 14.3$ & $41.8 \pm 13.6$ & $44.7 \pm 16.3$ & 0.180 \\
\hline$<55$ & $189(80.4)$ & $154(84.6)$ & $35(66.0)$ & \\
\hline $55-70$ & $38(16.2)$ & $23(12.6)$ & $15(28.3)$ & \\
\hline$>70$ & $8(3.4)$ & $5(2.8)$ & $3(5.7)$ & \\
\hline Female (\%) & $192(81.7)$ & $153(84.1)$ & $39(73.6)$ & 0.082 \\
\hline BMI $\left(\mathrm{kg} / \mathrm{m}^{2}\right)$ & $22.1 \pm 3.8$ & $22.9 \pm 3.8$ & $22.9 \pm 3.6$ & 0.925 \\
\hline ATA risk (\%) & & & & 0.013 \\
\hline Low & $124(52.8)$ & $105(57.7)$ & $19(35.8)$ & \\
\hline Intermediate & $42(17.8)$ & $31(17.0)$ & $11(20.8)$ & \\
\hline High & $69(29.4)$ & $46(25.3)$ & $23(43.4)$ & \\
\hline Size $(\mathrm{cm})$ & $2.3 \pm 1.4$ & $2.2 \pm 1.3$ & $2.7 \pm 1.7$ & 0.031 \\
\hline$\leq 1$ & $50(21.4)$ & $42(23.1)$ & $8(15.4)$ & \\
\hline$>1-2$ & $76(32.0)$ & $57(31.3)$ & $19(34.6)$ & \\
\hline$>2-4$ & $90(38.5)$ & $72(39.6)$ & $18(34.6)$ & \\
\hline$>4$ & $19(8.1)$ & $11(6.0)$ & $8(15.4)$ & \\
\hline Extrathyroidal extension (\%) & $18(7.7)$ & $12(6.6)$ & $6(11.5)$ & 0.238 \\
\hline $8^{\text {th }}$ AJCC staging (\%) & & & & $<0.001$ \\
\hline I & $211(89.7)$ & $171(94.0)$ & $40(75.4)$ & \\
\hline II & $19(8.1)$ & $10(5.5)$ & $9(17.0)$ & \\
\hline III & $2(0.9)$ & 0 & $2(3.8)$ & \\
\hline IV & $3(1.3)$ & $1(0.5)$ & $2(3.8)$ & \\
\hline Follow-up time (years) & $9.5 \pm 7.7$ & $9.4 \pm 7.6$ & $9.7 \pm 7.8$ & 0.769 \\
\hline Coexistence of Hashimoto's thyroiditis (\%) & $46(19.6)$ & $44(24.2)$ & $2(3.8)$ & $<0.001$ \\
\hline
\end{tabular}

AJCC: the American Joint Committee on Cancer.

3.2. Follow-Up and Clinical Response Status after Initial Treatment. Most patients had an excellent response $(n=151,64.3 \%)$. The biochemical incomplete and structural incomplete response rates were $17.0 \%(n=40)$ and $8.5 \%$ $(n=20)$, respectively. After completing initial treatment, 53 patients (22.6\%) developed persistent/recurrent cancer during the follow-up period. Overall disease-free survival was $77.4 \%$. A low mortality rate was observed in 11 patients $(4.7 \%)$ who died during the study period. Only four patients (1.7\%) died from a cancer-specific cause.
3.3. Factors Associated with Persistent and Recurrent Disease. Table 3 summarizes the potential risk factors of various clinicopathological characteristics on persistence and recurrence of cancer. Univariate analysis showed that age $\geq 55$ years, high ATA risk, and tumor size $>4 \mathrm{~cm}$ were associated with an increased risk of recurrence/persistence of cancer, whereas gender, BMI $>27 \mathrm{~kg} / \mathrm{m}^{2}$, multifocality, and extrathyroidal extension had no effects. In multivariate analysis, only age $\geq 55$ years was a significant predictor of a poor outcome. Further analysis revealed that higher recurrent rate 
TABle 2: Demographic data of papillary thyroid cancer in each decade of the study period.

\begin{tabular}{|c|c|c|c|c|}
\hline & $1987-1996(n=46)$ & $1997-2006(n=64)$ & $2007-2019(n=125)$ & $P$ value \\
\hline Age at initial diagnosis (years) & $38.6 \pm 13.2$ & $43.6 \pm 15.2$ & $43.3 \pm 14.0$ & 0.128 \\
\hline Female (\%) & $41(89.1)$ & $48(75.0)$ & $103(82.4)$ & 0.160 \\
\hline BMI $\left(\mathrm{kg} / \mathrm{m}^{2}\right)$ & $22.3 \pm 3.2$ & $23.1 \pm 3.9$ & $23.0 \pm 3.9$ & 0.502 \\
\hline ATA risk (\%) & & & & 0.036 \\
\hline Low & $29(63.1)$ & $37(57.8)$ & $58(46.4)$ & \\
\hline Intermediate & $11(23.9)$ & $7(10.9)$ & $24(19.2)$ & \\
\hline High & $6(13.0)$ & $20(31.3)$ & $43(34.4)$ & \\
\hline $8^{\text {th }}$ AJCC staging (\%) & & & & 0.597 \\
\hline I & $43(93.5)$ & $57(89.1)$ & $111(88.8)$ & \\
\hline II & $3(6.5)$ & $5(7.8)$ & $11(8.8)$ & \\
\hline III & 0 & 0 & $2(1.6)$ & \\
\hline IV & 0 & $2(3.1)$ & $1(0.8)$ & \\
\hline Coexistence of Hashimoto's thyroiditis (\%) & $7(15.2)$ & $10(15.6)$ & $29(23.2)$ & 0.328 \\
\hline PTMC (\%) & $7(15.2)$ & $12(19.0)$ & $31(24.8)$ & 0.348 \\
\hline
\end{tabular}

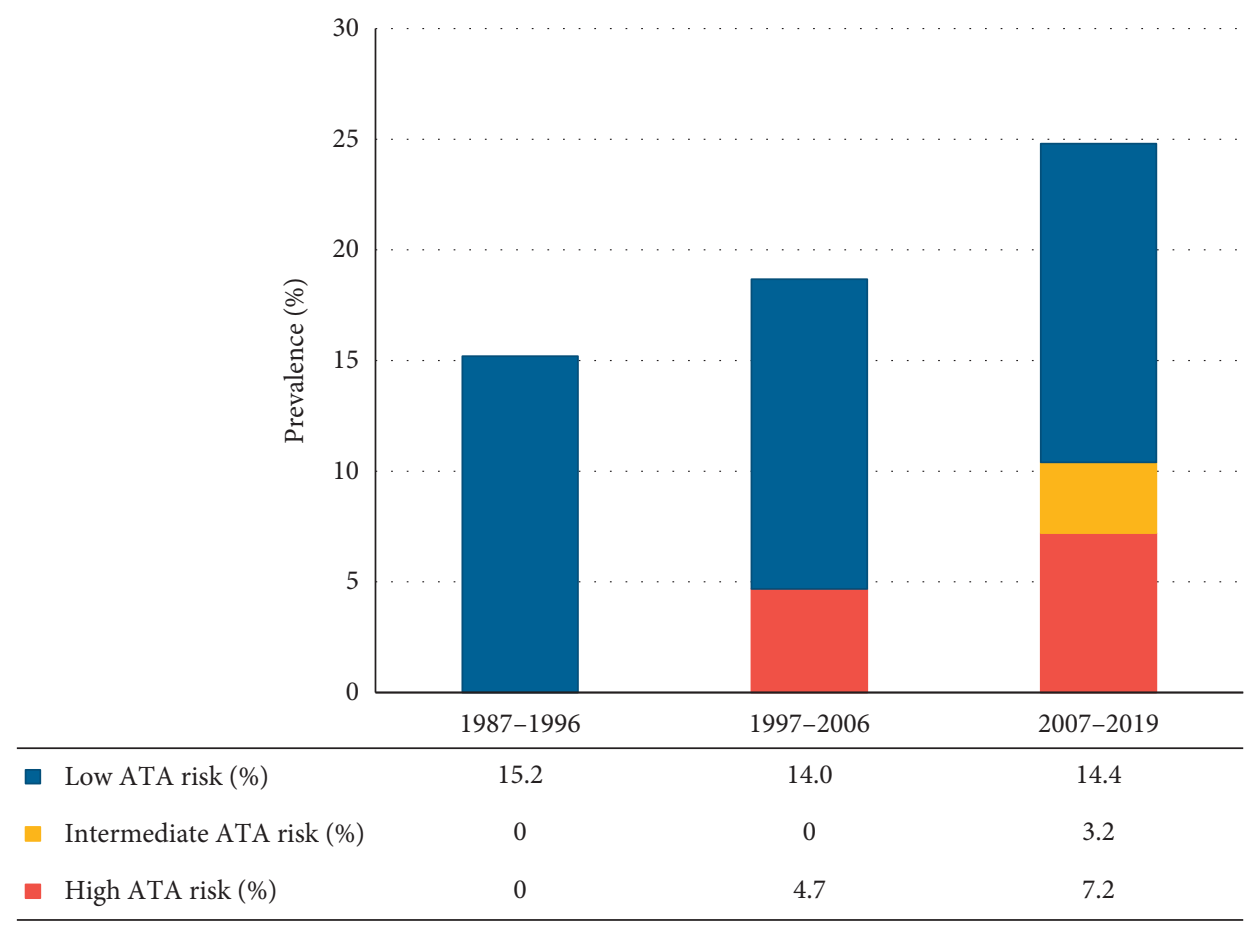

FIGURE 2: The prevalence of papillary thyroid microcarcinoma classified by ATA risk in each decade of the study period.

TABle 3: Potential factors of persistent/recurrent papillary thyroid cancer.

\begin{tabular}{|c|c|c|c|c|c|c|}
\hline \multirow[b]{2}{*}{ Factor } & \multicolumn{3}{|c|}{ Univariate analysis } & \multicolumn{3}{|c|}{ Multivariate analysis } \\
\hline & HR & $95 \% \mathrm{CI}$ & $P$ value & HR & $95 \% \mathrm{CI}$ & $P$ value \\
\hline Age $\geq 55$ years & 2.83 & $1.41-5.68$ & 0.003 & 2.67 & $1.27-5.61$ & 0.010 \\
\hline Male & 1.89 & $0.91-3.92$ & 0.086 & & & \\
\hline High ATA risk & 2.27 & $1.20-4.29$ & 0.012 & 1.73 & $0.86-3.45$ & 0.122 \\
\hline Tumor size $>4 \mathrm{~cm}$ & 3.24 & $1.45-7.22$ & 0.004 & 2.16 & $0.91-5.12$ & 0.081 \\
\hline Coexistence of Hashimoto's thyroiditis & 0.12 & $0.03-0.53$ & 0.005 & 0.16 & $0.04-0.68$ & 0.013 \\
\hline $\mathrm{BMI}>27 \mathrm{~kg} / \mathrm{m}^{2}$ & 0.84 & $0.34-2.04$ & 0.696 & & & \\
\hline Multifocality & 1.52 & $0.78-2.98$ & 0.219 & & & \\
\hline Extrathyroidal extension & 1.84 & $0.66-5.19$ & 0.244 & & & \\
\hline
\end{tabular}

was found only in patients at age $\geq 55$ years in the high-risk ATA group when compared to patients $<55$ years old $(P=0.001)$. This effect was not found in patients with low and intermediate ATA risk groups $(P=0.270$ and 0.051 , respectively). Coexistence of HT was revealed to be a protective factor in both univariate and multivariate analyses. 


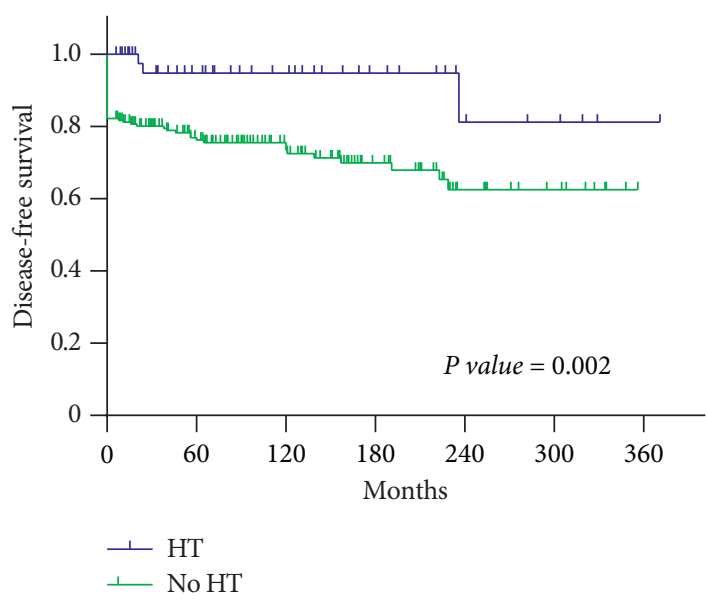

(a)

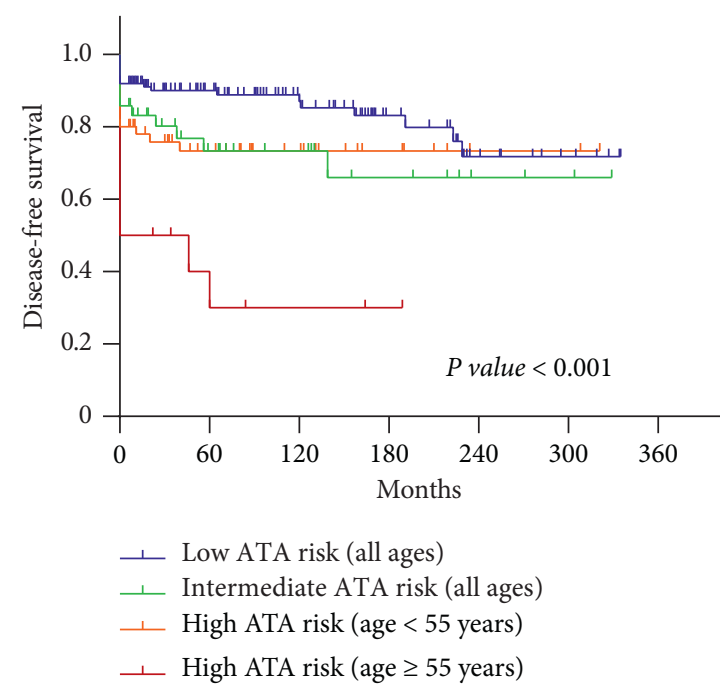

(b)

Figure 3: Disease-free survival curves of patients with papillary thyroid cancer based on the following classifications: (a) coexistence of Hashimoto's thyroiditis (HT); (b) ATA risk category.

Supplementary 1 shows the characteristics of PTC according to the coexistence of HT. Disease-free survival curves of the HT and ATA risk category are shown in Figure 3.

\section{Discussion}

Our main finding consistently showed that the prevalence rate of thyroid cancer has risen, particularly in the past decade due to an increase in the incidence of PTMC. A recent study reported that PTMC contributed to $30 \%$ of all cases of PTC [1], supported our finding (24.8\%). An increase in incidental findings from imaging and the need for a diagnosis have contributed to this observation. Moreover, genetic mutation and carcinogenesis from an increase in radiation exposure, dietary changes, and the use of chemical fertilizers or genetically modified food may also be responsible [14]. However, recurrence and mortality rates have been growing despite early diagnosis and treatment. A recent large cohort study showed that the mortality rate of thyroid cancer has sharply increased during the past decade [1]. Possible explanations may include an underestimation of the aggressiveness of the cancer from under-risk stratification or mutation of the tumor.

According to current guidelines, PTMC has a good prognosis. However, a previous study conducted during the early 2000 s reported that $14 \%$ of PTMCs were aggressive [15]. More recent study in 2019 indicated that up to $19 \%$ of PTMCs had advanced features, including lymph node metastasis, extrathyroidal extension, lymphovascular invasion, and distant metastasis [5]. These trends and clinical findings were similar to those found in our study. The $8^{\text {th }}$ edition AJCC guidelines do not suggest fine needle aspiration for PTMCs unless they have clear evidence of aggressive behavior and highly suspicious ultrasonography findings. This recommendation was based on the data from 1940 to 2000, which stated that PTMC had an excellent prognosis and a very low rate of recurrence (2-6\%) [16]. However, regarding the upward trend of PTMC aggressiveness, an individual tailored approach to treatment is essential.

Older age and recurrent rate have found a linear correlation. Chereau et al. indicated worse prognosis with increasing age, especially in patients $>75$ years old. The recurrence rate increased almost twofold in patients $>75$ years old compared to patients $<65$ years old ( $6.2 \%$ vs. $11.7 \%$, respectively) [17]. In addition, a recent study by Kauffmann et al. showed that older patients had a higher five-year mortality rate (hazard ratio $=2.3$ ) compared to patients $<45$ years old. This effect was independent of gender, race, number of comorbidities, type of operation, hospital volume, or insurance coverage [18]. However, previous study showed that the ATA risk category applying with age showed differences in survival [19]. Similar to our results, only high-risk ATA category with age at cutoff 55 years showed significantly higher recurrent rate, while this effect was not found in low and intermediate ATA risk category. Therefore, very old patients should be considered high-risk patients, and age should be applied with the ATA risk category to improve the stratification system.

Our study showed that patients with PTC and coexisting HT had favorable outcomes. HT has long been debated whether it is a risk factor for thyroid cancer and contribution of the prognosis [20]. It is believed that chronic inflammation of the thyroid gland and high TSH levels, typically found in patients with HT, might be associated with neoplastic changes [21, 22]. Several observational studies and meta-analyses have shown that HT was associated with PTC incidence [23-25] and better prognosis regarding less lymph node involvement, less extrathyroidal extension, smaller tumor size, and longer survival $[25,26]$. In a large retrospective study with a nine-year follow-up, the cancer-specific mortality and recurrence rates were lower in patients 
with coexisting PTC and HT compared to those without HT ( $2.2 \%$ vs. $4.6 \%$ and $4.3 \%$ vs. $14 \%$, respectively) [27]. Tumor cells can trigger both innate and noninnate immunity, which may lead to an antineoplastic immune response [28].

Recurrence/persistence of PTC has been reported to range from $8.4 \%$ to $32 \%$ [29], which was similar to our results. These variations might result from different initial approach methods, severity of the disease, treatment, race, and follow-up duration. In this study, most of our patients had a serum TSH level $<0.01$ over the treatment period which might affect the treatment outcomes.

4.1. Limitation of the Study. Our study has some limitations because of its retrospective nature and possible selection bias. Additionally, the data might not be fully representative of the total population because of the limited sample size. However, this study provides the trends and clinical characteristics of PTC over a 30-year period.

\section{Conclusions}

The incidence and aggressiveness of thyroid cancer have increased, and increased incidence of PTMC has contributed to this trend. The outcome of PTMC may not be favorable. Treatment should be in accordance with risk stratification. Coexistence with HT is considered a good prognostic, and age $\geq 55$ years is associated with poorer outcomes.

\section{Data Availability}

The data that support the findings of this study are restricted to the Institutional Review Board of Theptarin Hospital. Due to the privacy of patients, the data are available from the corresponding author upon reasonable request.

\section{Disclosure}

Parts of this manuscript had previously been presented as a poster in Endocrine Society Conference 2021 (virtual meeting). The funder had no role in the study design, data collection, and analysis.

\section{Conflicts of Interest}

The authors declare that there are no conflicts of interest regarding the publication of this paper.

\section{Acknowledgments}

This research would not have been possible without the support of all the staff in the research unit. The authors also thank the staff of Theptarin Hospital for their assistance. This work was supported by the Theptarin Research Unit, Theptarin Hospital (Grant no. 2/2019).

\section{Supplementary Materials}

Supplementary 1: demographic data of 235 papillary thyroid cancer patients according to the coexistence of HT. (Supplementary Materials)

\section{References}

[1] H. Lim, S. S. Devesa, J. A. Sosa, D. Check, and C. M. Kitahara, "Trends in thyroid cancer incidence and mortality in the United States, 1974-2013," JAMA, vol. 317, no. 13, pp. 1338-1348, 2017.

[2] R. L. Siegel, K. D. Miller, and A. Jemal, "Cancer statistics, 2020," CA: A Cancer Journal for Clinicians, vol. 70, no. 1, pp. 7-30, 2020.

[3] C. La Vecchia and E. Negri, "The thyroid cancer epidemicoverdiagnosis or a real increase?" Nature Reviews Endocrinology, vol. 13, no. 6, pp. 318-319, 2017.

[4] C. Durante, T. Montesano, M. Torlontano et al., "Papillary thyroid cancer: time course of recurrences during postsurgery surveillance," The Journal of Clinical Endocrinology \& Metabolism, vol. 98, no. 2, pp. 636-642, 2013.

[5] Z. Al-Qurayshi, N. Nilubol, R. P. Tufano, and E. Kandil, "Wolf in sheep's clothing: papillary thyroid microcarcinoma in the US," Journal of the American College of Surgeons, vol. 230, no. 4, pp. 484-491, 2020.

[6] B. R. Haugen, E. K. Alexander, K. C. Bible et al., "American thyroid association management guidelines for adult patients with thyroid nodules and differentiated thyroid cancer: the American thyroid association guidelines task force on thyroid nodules and differentiated thyroid cancer," Thyroid, vol. 26, no. 1, pp. 1-133, 2015.

[7] C. Zhang, Y. Li, J. Li, and X. Chen, "Total thyroidectomy versus lobectomy for papillary thyroid cancer: a systematic review and meta-analysis," Medicine (Baltimore), vol. 99, no. 6, e pages, Article ID e19073, 2020.

[8] A. Magreni, D. V. Bann, J. R. Schubart, and D. Goldenberg, "The effects of race and ethnicity on thyroid cancer incidence," JAMA Otolaryngology-Head \& Neck Surgery, vol. 141, no. 4, pp. 319-323, 2015.

[9] B. R. Shah, R. Griffiths, and S. F. Hall, "Thyroid cancer incidence among Asian immigrants to Ontario, Canada: a population-based cohort study," Cancer, vol. 123, no. 17, pp. 3320-3325, 2017.

[10] H. Jin, P. S. Pinheiro, J. Xu, and A. Amei, "Cancer incidence among Asian American populations in the United States, 2009-2011," International Journal of Cancer, vol. 138, no. 9, pp. 2136-2145, 2016.

[11] L. H. Kus, M. Shah, S. Eski, P. G. Walfish, and J. L. Freeman, "Thyroid cancer outcomes in Filipino patients," Archives of Otolaryngology-Head \& Neck Surgery, vol. 136, no. 2, pp. 138-142, 2010.

[12] R. M. Tuttle, B. Haugen, and N. D. Perrier, "Updated American Joint committee on cancer/tumor-node-metastasis staging system for differentiated and anaplastic thyroid cancer (eighth edition): what changed and why?" Thyroid, vol. 27, no. 6, pp. 751-756, 2017.

[13] Z. Al-Qurayshi, R. Robins, A. Hauch, G. W. Randolph, and E. Kandil, "Association of surgeon volume with outcomes and cost savings following thyroidectomy," JAMA OtolaryngologyHead \& Neck Surgery, vol. 142, no. 1, pp. 32-39, 2016.

[14] R. Vigneri, P. Malandrino, and M. Russo, "Is thyroid cancer increasing in incidence and aggressiveness?" The Journal of Clinical Endocrinology and Metabolism, vol. 105, no. 7, 2020.

[15] S. Noguchi, H. Yamashita, S. Uchino, and S. Watanabe, "Papillary microcarcinoma," World Journal of Surgery, vol. 32, no. 5, pp. 747-753, 2008.

[16] I. D. Hay, "Management of patients with low-risk papillary thyroid carcinoma," Endocrine Practice, vol. 13, no. 5, pp. 521-533, 2007. 
[17] N. Chereau, C. Trésallet, S. Noullet et al., "Prognosis of papillary thyroid carcinoma in elderly patients after thyroid resection: a retrospective cohort analysis," Medicine (Baltimore), vol. 95, no. 47, Article ID 5450, 2016.

[18] R. M. Kauffmann, J. B. Hamner, P. H. G. Ituarte, and J. H. Yim, "Age greater than 60 years portends a worse prognosis in patients with papillary thyroid cancer: should there be three age categories for staging?" BMC Cancer, vol. 18, no. 1, p. 316, 2018.

[19] P. Trimboli, A. Piccardo, A. Signore et al., "Patient Age is an independent risk factor of relapse of differentiated thyroid carcinoma and improves the performance of the American thyroid association stratification system," Thyroid, vol. 30, no. 5, pp. 713-719, 2020.

[20] R. H. Grogan, S. P. Kaplan, H. Cao et al., "A study of recurrence and death from papillary thyroid cancer with 27 years of median follow-up," Surgery, vol. 154, no. 6, pp. 1436-1447, 2013.

[21] X. Lai, Y. Xia, B. Zhang, J. Li, and Y. Jiang, "A meta-analysis of Hashimoto's thyroiditis and papillary thyroid carcinoma risk," Oncotarget, vol. 8, no. 37, pp. 62414-62424, 2017.

[22] R. Vita, A. Ieni, G. Tuccari, and S. Benvenga, "The increasing prevalence of chronic lymphocytic thyroiditis in papillary microcarcinoma," Reviews in Endocrine and Metabolic Disorders, vol. 19, no. 4, pp. 301-309, 2018.

[23] F. Boi, F. Pani, and S. Mariotti, "Thyroid autoimmunity and thyroid cancer: review focused on cytological studies," $E$ uropean Thyroid Journal, vol. 6, no. 4, pp. 178-186, 2017.

[24] I. S. Lee, A. T. Hsieh, T. W. Lee, T. I. Lee, and Y. M. Chien, "The association of thyrotropin and autoimmune thyroid disease in developing papillary thyroid cancer," International Journal of Endocrinology, vol. 2017, Article ID 5940367, , 2017.

[25] J.-H. Lee, Y. Kim, J.-W. Choi, and Y.-S. Kim, “The association between papillary thyroid carcinoma and histologically proven Hashimoto's thyroiditis: a meta-analysis," European Journal of Endocrinology, vol. 168, no. 3, pp. 343-349, 2013.

[26] L. Zhang, H. Li, Q. H. Ji et al., "The clinical features of papillary thyroid cancer in Hashimoto's thyroiditis patients from an area with a high prevalence of Hashimoto's disease," BMC Cancer, vol. 12, Article ID 610, 2012.

[27] B.-Y. Huang, C. Hseuh, T.-C. Chao, K.-J. Lin, and J.-D. Lin, "Well-differentiated thyroid carcinoma with concomitant Hashimoto's thyroiditis present with less aggressive clinical stage and low recurrence," Endocrine Pathology, vol. 22, no. 3, pp. 144-149, 2011.

[28] G. Graceffa, R. Patrone, S. Vieni et al., "Association between Hashimoto's thyroiditis and papillary thyroid carcinoma: a retrospective analysis of 305 patients," BMC Endocrine Disorders, vol. 19, no. 1, p. 26, 2019.

[29] G. W. Randolph, Q.-Y. Duh, K. S. Heller et al., "The prognostic significance of nodal metastases from papillary thyroid carcinoma can be stratified based on the size and number of metastatic lymph nodes, as well as the presence of extranodal extension," Thyroid, vol. 22, no. 11, pp. 1144-1152, 2012. 